\title{
Spectral and geometrical variation of the bidirectional reflectance distribution function of diffuse reflectance standards
}

\author{
Alejandro Ferrero, * Ana María Rabal, Joaquín Campos, \\ Alicia Pons, and María Luisa Hernanz \\ Instituto de Óptica, Consejo Superior de Investigaciones Científicas (CSIC), Madrid 28006, Spain \\ ${ }^{*}$ Corresponding author: alejandro.ferrero@csic.es
}

Received 17 September 2012; revised 7 November 2012; accepted 11 November 2012; posted 12 November 2012 (Doc. ID 176291); published 13 December 2012

\begin{abstract}
A study on the variation of the spectral bidirectional reflectance distribution function (BRDF) of four diffuse reflectance standards (matte ceramic, $\mathrm{BaSO}_{4}$, Spectralon, and white Russian opal glass) is accomplished through this work. Spectral BRDF measurements were carried out and, using principal components analysis, its spectral and geometrical variation respect to a reference geometry was assessed from the experimental data. Several descriptors were defined in order to compare the spectral BRDF variation of the four materials. (C) 2012 Optical Society of America
\end{abstract}

OCIS codes: $\quad 230.1980,290.1483$.

\section{Introduction}

The realization of diffuse reflectance and reflectance factor scales are based on diffuse reflection standards. Ideally, these standards should reproduce the perfectly reflecting diffuser (PRD), but actual standards only approach to this concept, since they neither reflect all incoming radiation nor are totally Lambertian. To assess the departure of actual diffuse reflection standards from PRD is important since different systematic errors might be related to it [1]. Therefore, the better the departure is characterize $\bar{d}$, the lower is the uncertainty of the measurements that use these reference standards, or the more accurate it can be accounted.

Various studies on the spectral reflectance distribution of the most common diffuse reflection standards have been published in literature for specific spectral ranges and illumination/viewing geometries [2-9]. However none has addressed the complete characterization within the visible range. Now, the development of more complex gonio-spectrophotometers

$1559-128 \mathrm{X} / 12 / 368535-06 \$ 15.00 / 0$

(C) 2012 Optical Society of America
[10-14] allows for spectral data at any measurement geometry to be obtained, so a most complete comparison of actual diffuse reflection standards to PRD can be done. The best way to do it is measuring their spectral bidirectional reflectance distribution function (BRDF) [15] because by definition PRD has got a constant $\mathrm{BRDF}$ value $\left(1 / \pi \mathrm{sr}^{-1}\right)$. This study has not completely been realized in previous jobs, so taking the advantage of the capabilities of a new goniospectrophotometer developed at Instituto de Optica in CSIC [10], able to carry out fast and accurate spectral BRDF measurements at any illumination/ viewing geometry, the BRDF of four important diffuse reflectance standards was measured and analyzed. Through this work, the spectral distribution variation of their BRDF is presented. The latter task was accomplished using principal components analysis (PCA), a multivariate mathematical procedure whose good performance on this kind of spectral BRDF data was proved previously $[16,17]$. PCA allows the BRDF to be expressed in a way where the spectral variation appears separated from geometrical variation, which simplifies the devising of a model and the data representation. 
The commercially available standards characterized in this work were: polished white Russian opal glass, used by NIST for its multi-angle white reflectance standards; Spectralon: sintered polytetrafluoroethylene (PTFE); matte ceramic reflection standard from CSS, pressed barium sulphate $\left(\mathrm{BaSO}_{4}\right)$ powder, prepared in our laboratory prior to the measurement (a molding was used, where the previously sieved $\mathrm{BaSO}_{4}$ was pressed). These materials are of primary interest since they are commonly used as diffuse reflection materials to transfer diffuse reflection or radiance factor $[\underline{8}, \underline{9}, \underline{18}, \underline{19}]$.

\section{Experimental Procedure and Data Analysis}

The gonio-spectrophotometer consists of a fixed, uniform, and collimated light source and a robot-arm to reposition the sample, making it possible to change automatically and simultaneously the illumination direction (defined by the polar $\theta$ and azimuth $\phi$ spherical coordinates, with subscripts $i$ ) and the viewing direction (with subscripts $s$ ). These spherical coordinates are defined relative to the sample coordinate system, whose $z$ axis is parallel to the sample's normal direction (Fig. 1). For the data acquisition a CS-2000 Konica Minolta spectroradiometer is used. This device operates within the visible range (380$780 \mathrm{~nm}$ ), performing spectral measurements with a $1 \mathrm{~nm}$ spectral sampling interval and a $4 \mathrm{~nm}$ bandwidth. As a result of the design, absolute and outof-incidence-plane spectral BRDF measurements of samples can be made in a fast and automatic way.

The illumination solid angle on the sample was almost collimated and defined by the diameter of a diaphragm $(2 \mathrm{~cm})$ and its distance to the sample $(113 \mathrm{~cm})$. Approximately the same solid angle was chosen for the viewing, using an aperture of $0.7 \mathrm{~cm}$ on front of the spectroradiometer. The illumination was $p$-polarized by a polarizer. It is important to specify the state of polarization, since it exists a dependence of the spectral BRDF on the polarization, the larger as more dominant are the reflections on the front surface (large incident angles and at specular directions). The uncertainty of the variable to be studied (relative spectral BRDF) is limited by the repeatability of the spectroradiometer $(0.6 \%)$ [10].

The spectral BRDF was sampled at geometries corresponding to combinations of $\theta_{i}$ and $\theta_{s}$ (which take

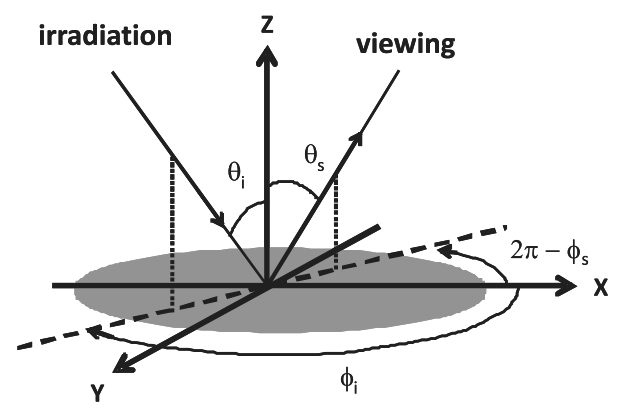

Fig. 1. Sample coordinate system showing spherical coordinates for illumination $\left(\theta_{i}, \phi_{i}\right)$ and viewing directions $\left(\theta_{s}, \phi_{s}\right)$. the values from $0^{\circ}$ to $75^{\circ}$ with steps of $5^{\circ}, \phi_{i}$ (which takes only the value $0^{\circ}$, assuming isotropy respect to the illumination direction) and $\phi_{s}$ (which takes the values $0^{\circ}$ and $180^{\circ}$, that is, the incidence plane).

The BRDF spectra $\left[f_{r}\left(\theta_{i}, \phi_{i} ; \theta_{s}, \phi_{s} ; \lambda\right)\right]$ were normalized with respect to the BRDF spectrum at the geometry $\left[\theta_{i}=0^{\circ}, \theta_{s}=45^{\circ}, \phi_{i}=0^{\circ}, \phi_{s}=180^{\circ}\right]$, which will be hereafter called the reference geometry:

$$
f_{r, \mathrm{rel}}\left(\theta_{i}, \phi_{i} ; \theta_{s}, \phi_{s} ; \lambda\right)=\frac{f_{r}\left(\theta_{i}, \phi_{i} ; \theta_{s}, \phi_{s} ; \lambda\right)}{f_{r}\left(0^{\circ}, 0^{\circ} ; 45^{\circ}, 180^{\circ} ; \lambda\right)} .
$$

As a consequence of this normalization, the measurement systematic errors are considerably reduced when assessing for the spectral distribution variation between spectra at different geometries.

For an ideal PRD, $f_{r \text {,rel }}$ would be the unity at any geometry and wavelength. In the case that the spectral distribution was invariant across the geometries, $f_{r \text { rel }}$ would be independent on the wavelength. As it will be shown in this work, this is not precisely the case in common material, being the main aim to characterize how much is this dependence on the wavelength. For this purpose, the spectral variation of $f_{r \text {,rel }}$ across the different geometries is studied by PCA.

According to the procedure described in [17], $f_{r, \text { rel }}$ can be expressed as

$$
\begin{aligned}
f_{r, \text { rel }}\left(\theta_{i}, \phi_{i} ; \theta_{s}, \phi_{s} ; \lambda\right)= & \left\langle f_{r, \text { rel }}\left(\theta_{i}, \phi_{i} ; \theta_{s}, \phi_{s} ; \lambda\right)\right\rangle_{\lambda} \\
& {\left[1+\sum_{j=1}^{M} c_{j}\left(\theta_{i}, \phi_{i} ; \theta_{s}, \phi_{s}\right) H_{j}(\lambda)\right] . }
\end{aligned}
$$

This equation corresponds to the multiplication of two factors: the spectral average of $f_{r}\left(\left\langle f_{r}\right\rangle_{\lambda}\right)$ with just geometrical dependence, and the sum of $M+1$ addends or components (within square brackets), being the first one the unity. Every addend is factorized in two: one containing the spectral information, $H_{j}(\lambda)$ (with null average value and a standard deviation value of 1 ), and another factor containing the geometrical information, $c_{j}\left(\theta_{i}, \phi_{i} ; \theta_{s}, \phi_{s}\right)$, that can be regarded as the weight of every spectral distribution at the different geometrical configuration and, according to the proposed formalism, as the relative variation of the spectral distribution from the reference geometry.

\section{Results and Discussion}

The results from the spectral BRDF measurement and the subsequent data processing is shown in Fig. 2 (matte ceramic reflection standard from CSS series), Fig. 3 (barium sulphate, $\mathrm{BaSO}_{4}$ ), Fig. $\underline{4}$ (Spectralon), and Fig. 5 (polished white Russian opal glass). In these figures, the half-plane containing the incident direction corresponds to negative $\theta_{s}$ values and the half-plane containing the specular direction corresponds to negative $\theta_{s}$ values. Every curve corresponds to a different $\theta_{i}$ value. For the sake of visibility, only $\theta_{i}=0^{\circ}$ and $\theta_{i}=75^{\circ}$ curves are labeled, the other 

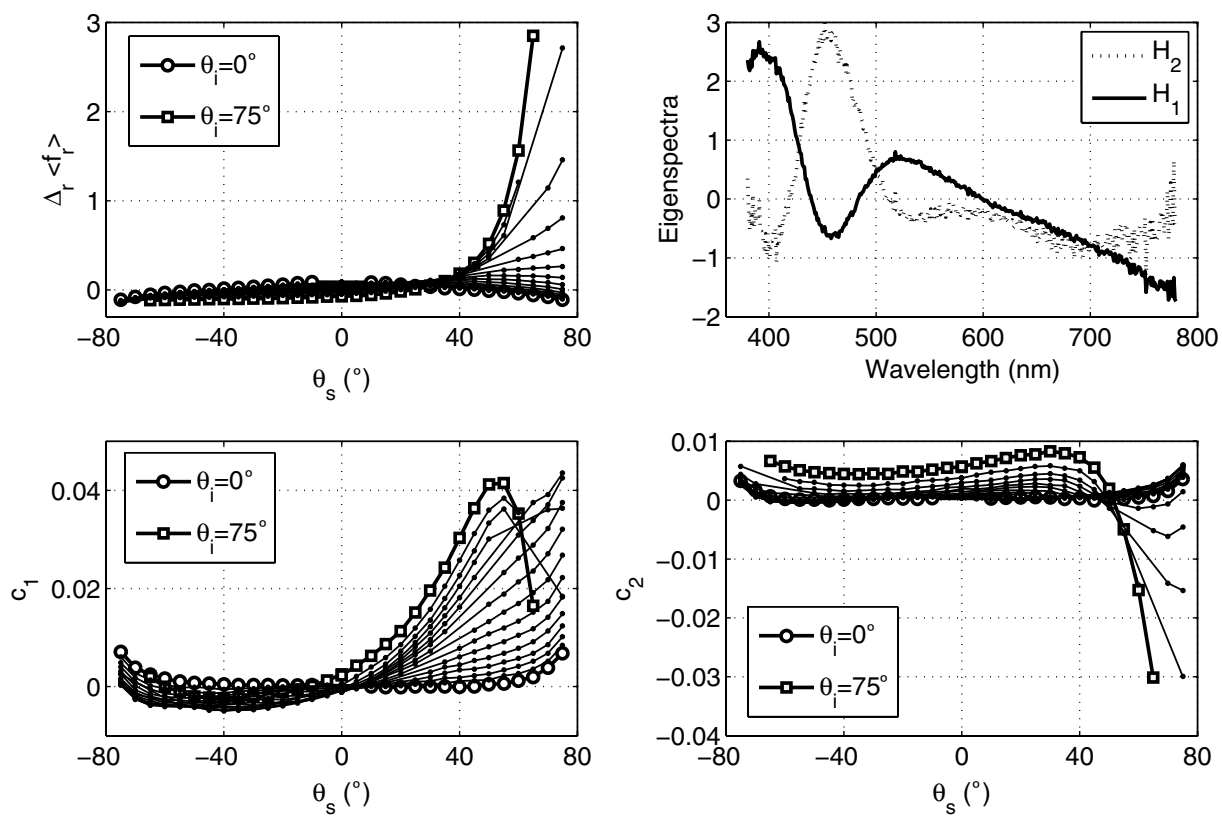

Fig. 2. Results on matte ceramic reflection standard from CSS series.

curves corresponding to intermediate $5^{\circ}$ intervals $\theta_{i}$ values. Specular geometries were not represented.

There are four plots in every figure. In the upper left plot, the relative variation of the wavelengthaveraged BRDF respect to the reference geometry (calculated as $\left.\Delta_{r} f_{r}=\left\langle f_{r \text {,rel }}\right\rangle_{\lambda}-1\right)$ is presented.

In the upper right plot, the first two eigenspectra $H_{j}(\lambda)$, which contribute the more to the spectral distribution variation, are shown (it should be reminded that the eigenspectra are sorted from the highest to the lowest contribution to the total variance).

In the bottom plots, the first two weighting coefficients $c_{1}$ (left) and $c_{2}$ (right) are represented, corresponding to the relative variation of the spectral distribution due to the components $\# 1\left[H_{1}(\lambda)\right]$ and $\# 2\left[H_{2}(\lambda)\right]$, respectively.

In addition, a set of descriptors are used in this work to better describe the deviation of BRDF's white standards from PRD. These descriptors are defined in Table 1 and identify variations in BRDF and in characteristic angles for every standard. Descriptors' numerical values obtained for every standard are shown in Table 2.

To facilitate the discussion, a distinction will be done between variations regarding Lambertian behavior and those regarding spectral changes.
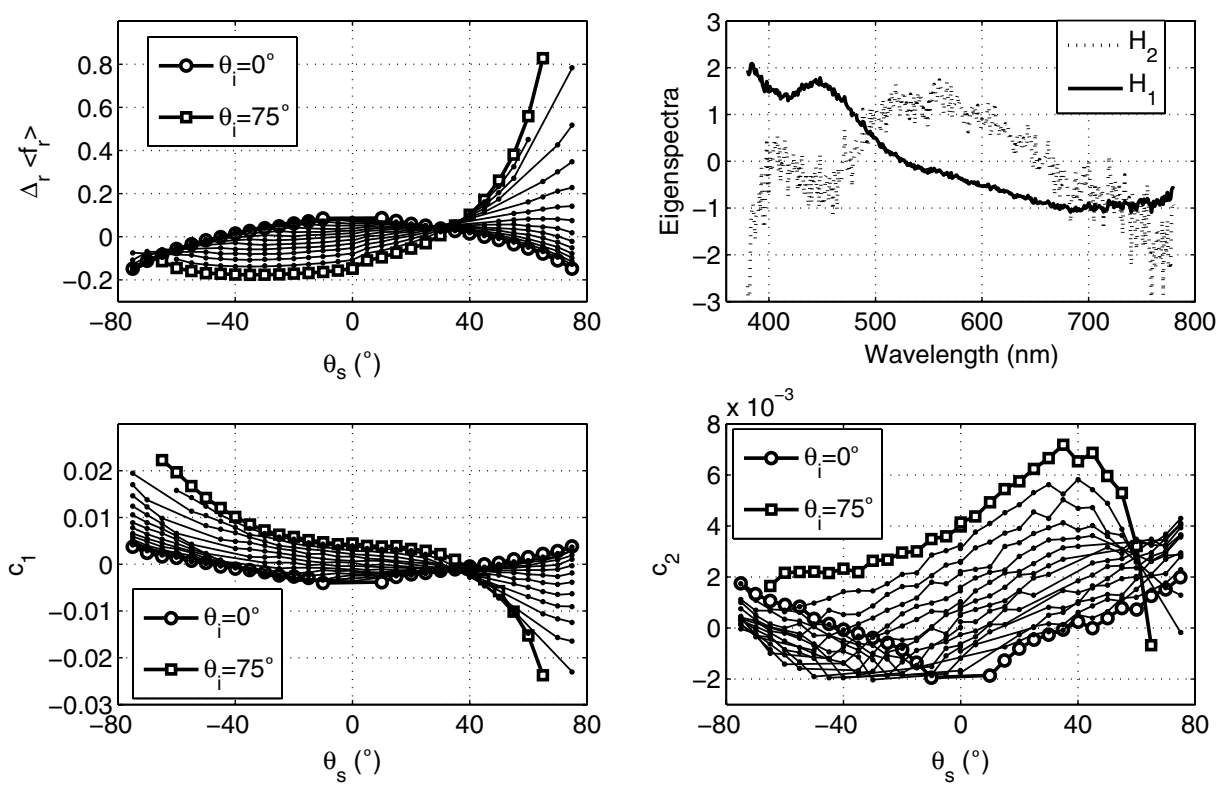

Fig. 3. Results on barium sulphate, $\mathrm{BaSO}_{4}$. 

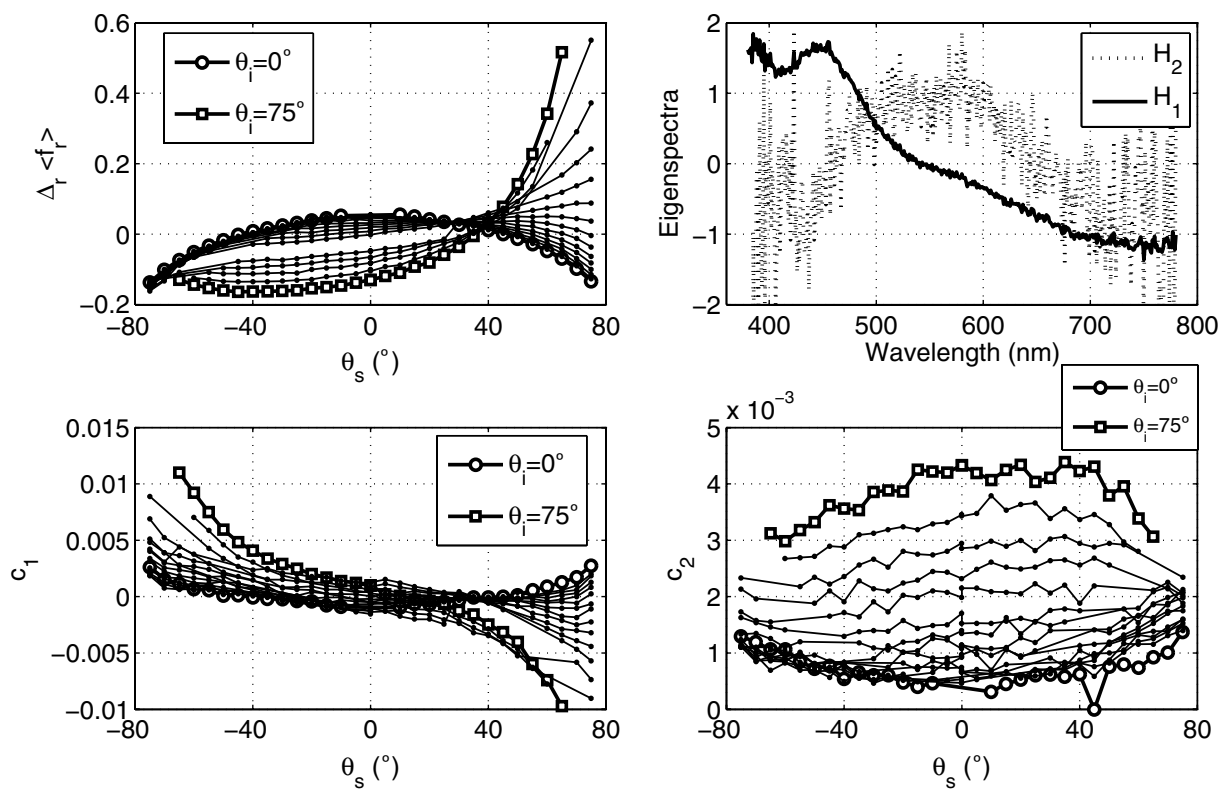

Fig. 4. Results on Spectralon.

\section{A. Lambertian behavior}

The graphs of the left upper corner show the BRDF of the four studied patterns is not flat like the one of the PRD. But a similar behavior can be observed for all the standards studied in this work. A curvature change on the BRDF as a function of the viewing angle is observed. For small angles of incidence curvature is negative, whereas for great angles of incidence, curvature is positive. The change is bigger in the half-plane containing the specular direction (positive values of $\theta_{s}$ in the graphs). This can be more clearly observed at the upper left plot in Fig. 4, because of the scale. Although not shown, this behavior is observed independently of the azimuthal angles $\left(\phi_{i}, \phi_{s}\right)$. Therefore, there may be an incidence angle,

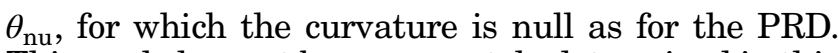
This angle has not been accurately determined in this job, but it is close to $50^{\circ}$ in the case of matte ceramic and Spectralon standards and close to $55^{\circ}$ in the case of $\mathrm{BaSO}_{4}$ and white Russian opal glass standards. These values are close to the $45^{\circ}$ reference geometry.

To quantify the maximum BRDF changes, descriptors $\delta_{i l}$ for low incidence angle, and $\delta_{\text {ih }}$ for high incidence angles are defined (see Table 2). For all studied standards $\delta_{\text {il }}$ lies between $15 \%$ and $20 \%$ (see Table 2), which makes no meaningful difference among them. However, the relative variation $\delta_{\text {ih }}$ at high illumination angles $\theta_{i}$ is very different among the standards (see Table 2). Matte ceramic presents the highest increase (almost 300\%), followed by
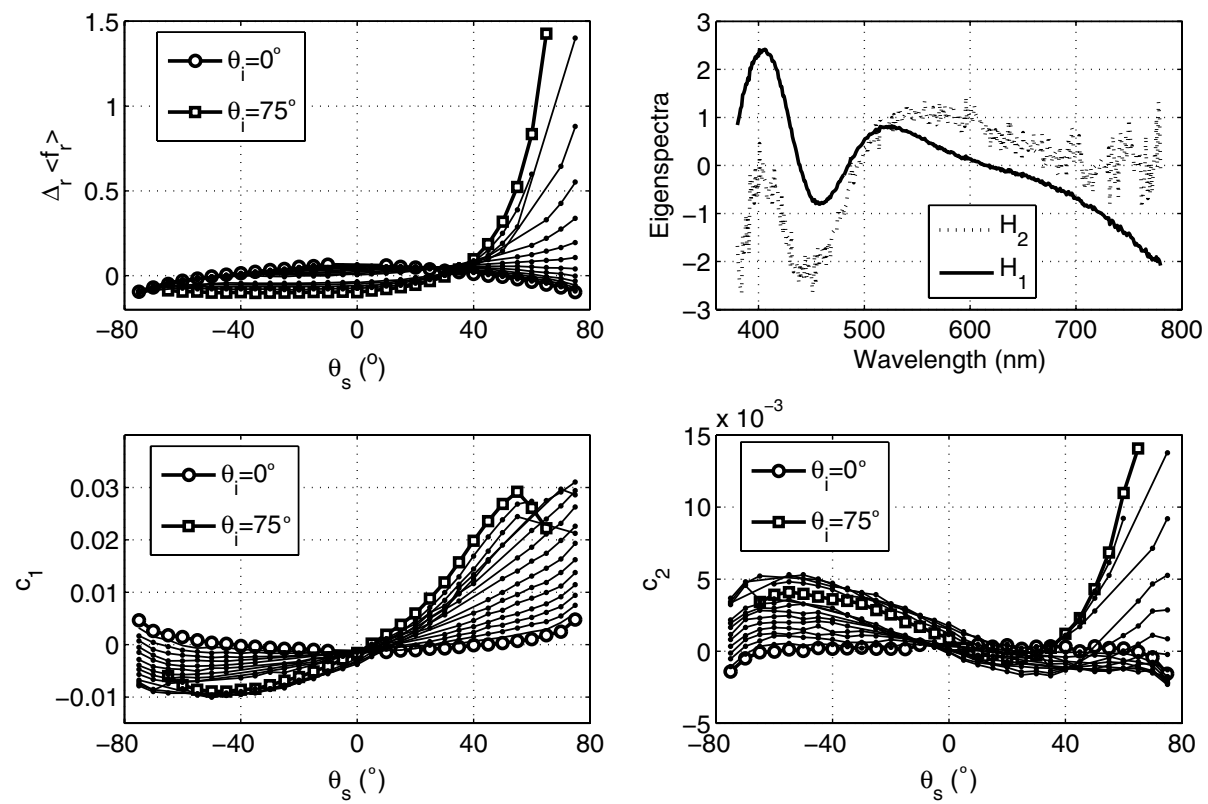

Fig. 5. Results on polished white Russian opal glass. 
Table 1. Definition of the Descriptors of the Spectral BRDF Relative Variation

\begin{tabular}{ll}
\hline Symbol & \multicolumn{1}{c}{ Definition } \\
\hline$\delta_{\mathrm{il}}$ & $\Delta_{r} f_{r}\left(10^{\circ}, 0^{\circ} ; 75^{\circ}, 180^{\circ}\right)-\Delta_{r} f_{r}\left(10^{\circ}, 0^{\circ} ; 0^{\circ}, 180^{\circ}\right)$ \\
$\delta_{\mathrm{ih}}$ & $\Delta_{r} f_{r}\left(65^{\circ}, 0^{\circ} ; 75^{\circ}, 180^{\circ}\right)-\Delta_{r} f_{r}\left(65^{\circ}, 0^{\circ} ; 0^{\circ}, 180^{\circ}\right)$ \\
$\delta_{\mathrm{sl}}$ & $\Delta_{r} f_{r}\left(75^{\circ}, 0^{\circ} ; 10^{\circ}, 180^{\circ}\right)-\Delta_{r} f_{r}\left(0^{\circ}, 0^{\circ} ; 10^{\circ}, 180^{\circ}\right)$ \\
$\delta_{\mathrm{sh}}$ & $\Delta_{r} f_{r}\left(75^{\circ}, 0^{\circ} ; 65^{\circ}, 180^{\circ}\right)-\Delta_{r} f_{r}\left(0^{\circ}, 0^{\circ} ; 65^{\circ}, 180^{\circ}\right)$ \\
$\left(\theta_{i, \text { ret }}, \theta_{s, \text { ret }}\right)$ & Geometry at $\phi_{i}=\phi_{s}$ where $\Delta_{r} f_{r}$ is minimum \\
$\delta_{\text {ret }}$ & $\Delta_{r} f_{r}\left(\theta_{i, \text { ret }}, 0^{\circ} ; 75^{\circ}, 0^{\circ}\right)-\Delta_{r} f_{r}\left(\theta_{i, \text { ret }}, 0^{\circ} ; \theta_{s, \text { ret }}, 0^{\circ}\right)$ \\
$\theta_{\text {nu }}$ & Maximum $\theta_{i}$ where $\Delta_{r} f_{r}\left(\theta_{i}, 0^{\circ} ; 75^{\circ}, 180^{\circ}\right)<$ \\
& $\Delta_{r} f_{r}\left(\theta_{i}, 0^{\circ} ; 45^{\circ}, 180^{\circ}\right)$ \\
$\theta_{\text {sp }, i(s), n}$ & Direction of illumination $($ or viewing $)$ where \\
& maximum spectral variation is produced due to the \\
& component \#n. \\
$\delta_{\mathrm{sp}, i(s), n}$ & Maximum spectral variation at constant $\theta_{\mathrm{sp}, i(s), n}$. \\
& Calculated as the difference between the maximum \\
& and minimum weights $\left(c_{n, \text { max }}-c_{n, \text { min }}\right)$ at $\theta_{\mathrm{sp}, i(s), n}$. \\
\hline
\end{tabular}

white Russian opal glass (around 150\%), $\mathrm{BaSO}_{4}$ (86\%) and, finally, Spectralon (63\%).

The minimum value of the wavelength-averaged $\mathrm{BRDF}$ is usually obtained in the half incident plane containing the illumination direction (negative angles in the graphs). Again two descriptors are used to describe this minimum: $\theta_{s, \text { ret }}$ for the direction at which the minimum is found and $\delta_{s \text { ret }}$ for the variation of the wavelength-averaged $\mathrm{BRDF}$ at the minimum with respect to the reference BRDF value. Two different cases were found in the standards studied:

- The minimum value lies in an intermediate viewing angle, which is the case of $\mathrm{BaSO}_{4}$ and Spectralon, whose $\theta_{s \text {,ret }}$ are $35^{\circ}$ and $45^{\circ}$, respectively. The subsequent relative increase from $\theta_{s \text {,ret }}$ to $\theta_{s}=75^{\circ}$, accounted by the descriptor $\delta_{s, \text { ret }}$ are $6.4 \%$ for $\mathrm{BaSO}_{4}$ and $3.4 \%$ for Spectralon. The minimum values are obtained in both cases for the highest illumination angle studied in this work $\left(\theta_{i \text {,ret }}=75^{\circ}\right)$.

- The minimum value lies in the highest viewing angle studied $\left(\theta_{s \text {,ret }}=75^{\circ}\right)$. It is the case of matte ceramic and white Russian opal glass.

Table 2. Descriptors of the Spectral BRDF Relative Variation for the White Reflectance Standards Matte Ceramic, $\mathrm{BaSO}_{4}$, Spectralon, and White Russian Opal Glass

\begin{tabular}{|c|c|c|c|c|}
\hline & Matte Ceramic & $\mathrm{BaSO}_{4}$ & Spectralon & Opal Glass \\
\hline$\delta_{\mathrm{il}}$ & -0.18 & -0.20 & -0.17 & -0.15 \\
\hline$\delta_{\text {ih }}$ & 2.74 & 0.86 & 0.63 & 1.46 \\
\hline$\delta_{\mathrm{sl}}$ & -0.14 & -0.18 & -0.17 & -0.14 \\
\hline$\delta_{\mathrm{sh}}$ & 2.91 & 0.91 & 0.58 & 1.48 \\
\hline$\left(\theta_{i, \text { ret }}, \theta_{s, \text { ret }}\right)\left({ }^{\circ}\right)$ & $(50,75)$ & $(75,35)$ & $(75,45)$ & $(50,75)$ \\
\hline$\delta_{\text {ret }}$ & 0 & 0.064 & 0.034 & 0 \\
\hline$\theta_{\mathrm{nu}}\left({ }^{\circ}\right)$ & 50 & 55 & 50 & 55 \\
\hline$\delta_{\mathrm{sp}, i, 1}$ & 0.048 & 0.046 & 0.021 & 0.040 \\
\hline$\theta_{\mathrm{sp}, i, 1}\left({ }^{\circ}\right)$ & 55 & 75 & 75 & 55 \\
\hline$\delta_{\mathrm{sp}, i, 2}$ & 0.038 & 0.008 & 0.001 & 0.015 \\
\hline$\theta_{\mathrm{sp}, i, 2}\left({ }^{\circ}\right)$ & 75 & 75 & 75 & 75 \\
\hline$\delta_{\mathrm{sp}, s, 1}$ & 0.041 & 0.03 & 0.012 & 0.029 \\
\hline$\theta_{\mathrm{sp}, s, 1}\left({ }^{\circ}\right)$ & 55 & 75 & 75 & 55 \\
\hline$\delta_{\mathrm{sp}, s, 2}$ & 0.036 & 0.007 & 0.004 & 0.016 \\
\hline$\theta_{\mathrm{sp}, s, 2}\left({ }^{\circ}\right)$ & 75 & 35 & 45 & 75 \\
\hline
\end{tabular}

\section{B. Spectral behavior}

The spectral distribution varies depending on the measurement geometry. Eigenspectra $H_{1}(\lambda)$ and $H_{2}(\lambda)$ (upper right plots in Figs. 2-5), obtained from the PCA, modify the geometry-averaged spectral $\mathrm{BRDF}$ as a weighted combination that depends on the geometry (Eq. 2). Since $H_{1}(\lambda)$ and $H_{2}(\lambda)$ were normalized to their standard deviations, the weighting coefficients $c_{1}$ and $c_{2}$ represent the relative spectral variation with respect to the reference geometry, and the difference between the maximum and minimum weights at a specific illumination/viewing direction represent the total relative spectral variation at that direction. Again, using a descriptor for the incidence direction at which the maximum spectral variation is obtained $\left(\theta_{\mathrm{sp}, i, n}\right)$ and another for the maximum variation $\left(\delta_{\mathrm{sp}, i, n}\right)$, two different cases can be distinguished:

- The contribution of $H_{2}(\lambda)$ is negligible with respect to the contribution of $H_{1}(\lambda)$, which is the case of $\mathrm{BaSO}_{4}$ and Spectralon. In both materials, the highest spectral variation is produced at the highest incidence angle $\theta_{i}$ studied at this work $\left(\theta_{\mathrm{sp}, i, 1}=75^{\circ}\right)$, reaching values of $4.6 \%$ for $\mathrm{BaSO}_{4}$ and $2.1 \%$ for Spectralon.

- The contribution of $H_{2}(\lambda)$ is comparable to the contribution of $H_{1}(\lambda)$. In some specific geometries $H_{1}(\lambda)$ contribute more than $H_{2}(\lambda)$ and vice versa. This is the case of matte ceramic and white Russian opal glass. In both materials the relative spectral variation corresponding to $H_{1}(\lambda)$ is the highest at $\theta_{\mathrm{sp}, i, 1}=55^{\circ}$, reaching values of $4.8 \%$ for matte ceramic and $4 \%$ for white Russian opal glass. In both materials, the relative spectral variation corresponding to $H_{2}(\lambda)$ is the highest at $\theta_{\mathrm{sp}, i, 1}=75^{\circ}$, reaching values of $3.8 \%$ for matte ceramic and $1.5 \%$ for white Russian opal glass. The weighting coefficients of these eigenframes for every geometry are shown at the bottom plots of Figs. $\underline{2}-\underline{5}$, as mentioned before.

\section{Conclusions}

The variation of spectral BRDF of four common diffuse reflectance standards (matte ceramic, $\mathrm{BaSO}_{4}$, Spectralon, and white Russian opal glass) was measured and compared. The variation was reported with respect to a reference geometry $\left[\theta_{i}=0^{\circ}, \theta_{s}=45^{\circ}\right.$, $\phi_{i}=0^{\circ}, \phi_{s}=180^{\circ} \mathrm{]}$. Using $\mathrm{PCA}$, this variation can be expressed a linear combination of just two eigenspectra, with weighting coefficients depending on the geometry.

The four standards studied are not Lambertian since their BRDF is not a constant value. This result had already been shown for other authors, but the systematic study done in this work has shown that different behaviors can be observed. The curvature of the BRDF as a function of viewing angle changes with the angle of incidence. Furthermore the change is not the same for all the standards.

The matte ceramic reference material showed the highest spectral variation (5\%), but only slightly higher than the spectral variation of the $\mathrm{BaSO}_{4}$. The least spectral variation was found in the Spectralon 
(2\%). In any case, the spectral variation increases at the highest illumination angle on the sample studied in this work.

The authors are grateful to "Plan Nacional de Fsica" for funding this work (FIS2010-19756-E), and to the JAE Program from CSIC and "European Social Fund" for awarding us with a researcher in formation.

\section{References and Notes}

1. A. Pons, J. Campos, J. A. Méndez, and J. Martín, "Reflectance changes in white reflectance standards measured in different instruments with $0 / d$ geometry," Proceedings of 26th Session of the CIE, CIE 1.178:2007 (CIE, 2007), Vol. 1, pp. D2-46-D2-49.

2. W. Budde, "Standards of reflectance," J. Opt. Soc. Am. 50, 217-220 (1960)

3. F. W. Billmeyer, Jr., D. L. Lewis, and J. G. Davidson, "Goniophotometry of pressed barium sulfate," Color Eng. 9, 31-36 (1971).

4. W. Erb, "Requirements for reflection standards and the measurement of their reflection values," Appl. Opt. 14, 493-499 (1975).

5. Subcommittee "Standards and Techniques", "CIE 046-1979, A review of publications on properties and reflection values of material reflection standards" (CIE, 1979).

6. S. Holopainen, F. Manoocheri, E. Ikonen, K.-O. Hauer, and A. Höpe, "Comparison measurements of 0:45 radiance factor and goniometrically determined diffuse reflectance," Appl. Opt. 48, 2946-2956 (2009).

7. V. R. Weidner and Jack J. Hsia, "Reflection properties of pressed polytetrafluoroethylene powder," J. Opt. Soc. Am. 71, 856-861 (1981).

8. J. C. Zwinkels and W. Erb, "Comparison of absolute $d / 0$ diffuse reflectance factor scales of the NRC and the PTB," Metrologia 34, 357-363 (1997).

9. E. A. Early, P. Y. Barnes, B. C. Johnson, J. J. Butler, C. J. Bruegge, S. F. Biggar, P. S. Spyak, and M. M. Pavlov,
"Bidirectional reflectance round-robin in support of the earth observing system program," J. Atmos. Ocean. Technol. 17, 1077-1091 (2000).

10. A. M. Rabal, A. Ferrero, J. Campos, J. L. Fontecha, A. Pons, A. M. Rubiño, and A. Corróns, "Automatic goniospectrophotometer for the absolute measurement of the spectral BRDF at in\&out-of-plane and retroreflection geometries," Metrologia 49, 213-223 (2012).

11. T. A. Germer and C. C. Asmail, "Goniometric optical scatter instrument for out-of-plane ellipsometry measurements," Rev. Sci. Instrum. 70, 3688-3695 (1999).

12. D. Hünerhoff, U. Grusemann, and A. Höpe, "New robot-based gonioreflectometer for measuring spectral diffuse reflection," Metrologia 43, S11-S16 (2006).

13. F. B. Leloup, S. Forment, P. Dutré, M. R. Pointer, and P. Hanselaer, "Design of an instrument for measuring the spectral bidirectional scatter distribution function," Appl. Opt. 47, 5454-5467 (2008).

14. R. Baribeau, W. S. Neil, and E. Côté, "Development of a robotbased gonioreflectometer for spectral BRDF measurement," J. Mod. Opt. 56, 1497-1503 (2009).

15. F. E. Nicodemus, J. C. Richmond, and J. J. Hsia, "Geometrical considerations and nomenclature for reflectance," National Bureau of Standards Monograph 160 (U.S. Department of Commerce, 1977), 3-7.

16. A. Ferrero, J. Campos, A. M. Rabal, A. Pons, M. L. Hernanz, and A. Corróns, "Principal components analysis on the spectral bidirectional reflectance distribution function of ceramic colour standards," Opt. Express 19, 19199-19211 (2011).

17. A. Ferrero, J. Campos, A. M. Rabal, A. Pons, M. L. Hernanz, and A. Corróns, "Geometrical representation of the spectral bidirectional reflectance distribution function (BRDF) of pearlescent samples," J. Opt. Soc. Am. A 29, 842-847 (2012).

18. A. Springsteen, "Standards for the measurement of diffuse reflectance-an overview of available materials and measurement laboratories," Anal. Chim. Acta 380, 379-390 (1999).

19. A. Höpe and K.-O. Hauer, "Three-dimensional appearance characterization of diffuse standard reflection materials," Metrologia 47, 295-304 (2010). 